\title{
BIVARIATE PROBABILISTIC CONSTRAINED PROGRAMMING FOR INTERFERENCE EXPLOITATION IN THE COGNITIVE RADIO
}

\author{
*Ka Lung Law and *Christos Masouros and $\dagger$ Marius Pesavento \\ *Department of Electrical and Electronic Engineering, University College London, London, UK \\ $\nmid$ Communication Systems Group, Technische Universität Darmstadt, D-64283 Darmstadt, Germany \\ E-mail: lungkalaw@gmail.com, chris.masouros@ieee.org,mpesa@nt.tu-darmstadt.de
}

\begin{abstract}
In this paper, we study a constructive interference based cognitive radio beamforming optimization problem under perfect channel state information at the transmitter and the knowledge of data information. The beamformers are designed to minimize the worst secondary user's symbol error probability under constraints on the instantaneous total transmit power, and the power of the instantaneous interference in the primary link. The problem is formulated as a bivariate probabilistic constrained programming problem and can be solved using the barrier method. Our simulations indicate that the proposed technique offers a significantly improved performance over the conventional technique, while guaranteeing the quality of service (QoS) of primary users on an instantaneous basis, in contrast to the average QoS guarantees of conventional beamformers.
\end{abstract}

Index Terms - Downlink beamforming, robust design, symbol error probability, convex optimization, constructive interference.

\section{INTRODUCTION}

Cognitive radio (CR) schemes has provided an effective way to increase the radio resource utilization and spectral efficiency, by allowing the utilization of the licensed spectrum by secondary links [1-5]. In CR networks, the primary users (PUs) have the highest priority to access the spectrum without being aware of the existence of the unlicensed secondary user (SU) network. However, the PU network is willing to grant spectrum access to the SU network under the premise that the interference created by the secondary base station (SBS) does not exceed a predefined threshold [4].

As regards the CR transmission, the power minimization and signal to interference plus noise ratio (SINR) balancing problem for SUs with average interference power constraints of the primary users has been discussed in [5, 6]. Conventionally this problem is solved by (sequential) approximation as of second-order cone programs (SOCPs). To achieve more flexibility than that of the worst-case based design, channel outage univariate probabilistic constrained programming (UPSP) downlink beamforming problem has been developed [7, 8]. Nevertheless, the techniques of solving for UPSP problem could not be extended to multivariate probabilistic constrained programming problem as the problem is non-convex in general [9].

In order to improve the performance, the above mentioned SINR-based CR downlink beamforming problems are designed to mitigate the multiuser interference among the SUs. However, the associated drawback is that in SINR-based designs, some degrees of freedom in the beamforming design are used to suppress and eliminate the interference, which results in an overall increase of the transmitted power. This can be overcome by utilizing the knowledge of both channel state information (CSI) and SU's information symbols at the SBS to exploit the resulting interference in the secondary links. In this case beamformers can be designed to enhance the useful signal by steering the received signals, containing both the desired and the interfering signals, into the correct detection region instead of separately amplifying and suppressing the desired and the interfering signals, respectively [10-19]. This approach is also known as a constructive interference precoding.

In line with the above, this paper extends the work on the downlink beamforming optimization problem by exploiting the constructive interference [17, 18] to the CR scenarios where it was previously inapplicable. Since we do not have symbol information from PBS and by the law of large number, we assume the interference incorporated in the noise term is also a circularly symmetric complex Gaussian with zero mean. We assume that the phase-shift keying (PSK) modulation and the time division duplexing (TDD) protocol are applied, instantaneous CSI is available at the transmitter and instantaneous SU transmit data information are utilized at the SBS, as in [17, 18]. We formulate the beamformer design problem to minimize the worst SU's symbol error probability (WSUSEP) subject to total transmit power and PU instantaneous interference constraints, where WSUSEP is defined as the probability that worst SU wrongly decodes its symbol.

Notation: $\mathrm{E}\{\cdot\}, \operatorname{Pr}(\cdot),|\cdot|,\|\cdot\|,(\cdot)^{*}(\cdot)^{T}, \arg (\cdot), \bmod , \operatorname{Re}(\cdot)$ and $\operatorname{Im}(\cdot)$ denote the statistical expectation, the probability function, the absolute value, the Euclidean norm, the complex conjugate, and the transpose, the angle in a complex plane between the positive real axis to the line joining the point to the origin, the modulo operation, the real part, and the imaginary part, respectively.

\section{SYSTEM MODEL AND CONVENTIONAL DOWNLINK BEAMFORMING PROBLEM}

We consider a single cell CR system, which consists of a single $N$-antenna SBS, $K$ single-antenna SUs and $L$ single-antenna PUs. The signal transmitted by the SBS is given by the $N \times 1$ vector $\mathbf{x}=\sum_{k=1}^{K} \mathbf{w}_{i} b_{i}$, where $b_{i} \triangleq e^{j \vartheta_{i}}$ is the unit amplitude $M$-order PSK ( $M$-PSK) modulated symbol, $\vartheta_{i} \triangleq i \pi / M$ is the phase of the constellation point for $i$ th transmit data symbol, and $\mathbf{w}_{i}$ is the $N \times 1$ beamforming weight vector for the $i$ th SU. Let $\mathbf{h}_{i}$ be the $N \times 1$ channel vector from SBS to the $i$ th SU. The received signal of the $i$ th SU is $y_{i}=\mathbf{h}_{i}^{T} \mathbf{x}+n_{i}$ where $n_{i}$ at the $i$ th $\mathrm{SU}$ is a circularly symmetric complex Gaussian with zero mean. 


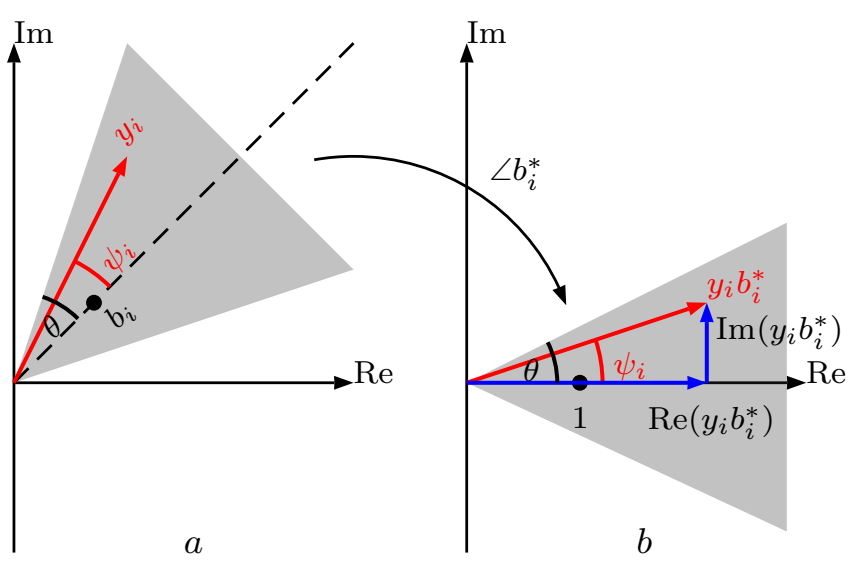

Fig. 1: In $M$-PSK, (a) constructive interference $y_{i}$ within correct detection region; (b) vector decomposition of $y_{i} b_{i}^{*}$ after rotation by $\angle b_{i}^{*}$.

\subsection{Max-Min Fair Problem}

The conventional SINR balancing CR downlink beamforming problem aims to maximize the minimum SINR subject to average interference and total transmitted power constraints. The problem can be written as [5]

$$
\begin{aligned}
\max _{\mathbf{w}_{i}, \gamma} \gamma \text { s.t. } & \frac{\left|\mathbf{h}_{i}^{T} \mathbf{w}_{i}\right|^{2}}{\sum_{\substack{j=1 \\
j \neq i}}^{K}\left|\mathbf{h}_{i}^{T} \mathbf{w}_{j}\right|^{2}+\sigma^{2}} \geq \gamma, i=1, \ldots, K, \\
& \sum_{i=1}^{K}\left\|\mathbf{w}_{i}\right\|^{2} \leq P_{0}, \sum_{i=1}^{K}\left|\mathbf{g}_{l}^{T} \mathbf{w}_{i}\right|^{2} \leq \epsilon_{l}, l=1, \ldots, L,
\end{aligned}
$$

where $\mathrm{g}_{l}$ is the $N \times 1$ channel vector between the SBS and $l$ th PU, $P_{0}$ is the total transmitted power budget and $\epsilon_{l}$ is the maximum admitted interference power caused by the SBS at the $l$ th PU, and $\sigma^{2}$ is the noise variance for all SUs. In [5]-[6], it is common to assume the independence of the symbols transmitted to different users, i.e., $\mathrm{E}\left\{b_{j}^{*} b_{i}\right\}=0$ for $i \neq j$. Problem (1b) can be transformed into a quasi-convex optimization problem and can be solved using the bisection method and sequential SOCP. Nevertheless, the above problem does not take instantaneous interference exploitation into account for the transmit data symbols as a part of the optimization problem for each transmission.

\section{WSUSEP-BASED CR DOWNLINK BEAMFORMING FOR INTERFERENCE EXPLOITATION}

\subsection{Constructive interference Exploitation}

With the aid of exploiting the instantaneous interference and adapting the beamformers, the constructive interference can alter the received signals further into the correct detection region to improve the system performance. Inspired by this idea, we provide a systematic treatment of constructive interference as illustrated in Fig. 11 a), where the nominal PSK constellation point is represented by the black circle. According to [17], we say that the received signal $y_{i}$ exploits the interference constructively if $y_{i}$ falls within the correct detection region, which is the shaded area shown in Fig. 1 a). Let $\psi_{i}$ in Fig. 11a) denote the angle between the received signal $y_{i}$ and the transmitted symbol $b_{i}$ in the complex plane. Note that the angle $\psi_{i}$ depends on the transmitted signal $\mathbf{x}$ and the noise $n_{i}$. Hence the angle $\psi_{i}$ can be treated as a function of $\mathbf{x}$ and $n_{i}$, i.e.,

$$
\begin{aligned}
\psi_{i}\left(\mathbf{x}, n_{i}\right) & =\left(\arg y_{i}-\arg b_{i}\right) \quad \bmod 2 \pi \\
& =\arg \left(y_{i} b_{i}^{*}\right)=\tan ^{-1}\left(\frac{\operatorname{Im}\left(y_{i} b_{i}^{*}\right)}{\operatorname{Re}\left(y_{i} b_{i}^{*}\right)}\right)
\end{aligned}
$$

where $\operatorname{Im}\left(y_{i} b_{i}^{*}\right)$ and $\operatorname{Re}\left(y_{i} b_{i}^{*}\right)$ are the projections of $y_{i} b_{i}^{*}$ onto the real and imaginary axis, respectively. The product $y_{i} b_{i}^{*}$ is displayed in Fig. 1 b) along with the corresponding decision region and the angle $\psi_{i}\left(\mathbf{x}, n_{i}\right)$. The received signal $y_{i}$ of the $i$-th user is detected correctly, if and only if

$$
\psi_{i}\left(\mathbf{x}, n_{i}\right) \quad \in \mathcal{A}_{-\theta}^{\theta}, \quad i=1, \ldots, K,
$$

where the angular set $\mathcal{A}_{\theta_{1}}^{\theta_{2}} \triangleq\left\{\tilde{\psi} \bmod 2 \pi \mid \theta_{1} \leq \tilde{\psi} \leq \theta_{2}, \tilde{\psi} \in \mathbb{R}\right\}$ defines the decision region and $\theta=\pi / M$ is the maximum angular shift for an M-PSK constellation. Based on above definition and discussion, we formulate in the following section the CR beamformer design to exploit the instantaneous interference.

\subsection{WSUSEP Approach}

In this section, we derive the WSUSEP-based CR downlink beamforming problem. The idea of this approach is to design the beamformers to steer the receive signals of SUs into the corresponding decision regions to reduce the corresponding symbol error. Furthermore, since the distribution of noise is known, we can calculate the symbol error probability (SEP) for each SU and use the WSUSEP as an objective function. The beamformer design minimizes the WSUSEP subject to the instantaneous total transmit power and instantaneous interference power constraints, which can be written as

$$
\begin{array}{cl}
\min _{\mathbf{x}, \rho} \rho \text { s.t. } & \operatorname{Pr}\left(\psi_{i}\left(\mathbf{x}, n_{i}\right) \in \mathcal{A}_{\theta}^{2 \pi-\theta}\right) \leq \rho, i=1, \ldots, K \\
& \|\mathbf{x}\|^{2} \leq P,\left|\mathbf{g}_{l}^{T} \mathbf{x}\right|^{2} \leq \epsilon_{l}, l=1, \ldots, L
\end{array}
$$

where $\rho$ models the WSUSEP, $\operatorname{Pr}\left(\psi_{i}\left(\mathbf{x}, n_{i}\right) \in \mathcal{A}_{\theta}^{2 \pi-\theta}\right)$ is $i$ th SU's SEP, i.e., the probability that the received signal falls outside the correct detection region and $\psi_{i}\left(\mathbf{x}, n_{i}\right) \notin \mathcal{A}_{-\theta}^{\theta},\|\mathbf{x}\|^{2}$ is the instantaneous total transmitted power from the SBS, and $\left|\mathbf{g}_{l}^{T} \mathbf{x}\right|^{2}$ is the instantaneous interference power for SBS to the $l$ th PU. By considering the complement of the symbol error set, 4a can be reformulated as

$$
1-\operatorname{Pr}\left(\psi_{i}\left(\mathbf{x}, n_{i}\right) \in \mathcal{A}_{-\theta}^{\theta}\right) \leq \rho .
$$

First let us simplify the set $\mathcal{A}_{-\theta}^{\theta}$, i.e., (3). By (2), the classification criteria (3) can be directly reformulated as the following alternatives: $\mathrm{I}: \frac{\left|\operatorname{Im}\left(y_{i} b_{i}^{*}\right)\right|}{\operatorname{Re}\left(y_{i} b_{i}^{*}\right)} \leq \tan \theta, \quad$ for $\operatorname{Re}\left(y_{i} b_{i}^{*}\right)>0, \mathrm{II}: y_{i} b_{i}^{*}=$ 0 , for $\operatorname{Re}\left(y_{i} b_{i}^{*}\right)=0$, which is equivalent to the single inequality

$$
\left|\operatorname{Im}\left(y_{i} b_{i}^{*}\right)\right|-\operatorname{Re}\left(y_{i} b_{i}^{*}\right) \tan \theta \leq 0 .
$$

In this paper, we only consider M-PSK modulation schemes with $M \geq 4$. Introducing the real-valued parameter vector representation $\overline{\mathbf{x}} \triangleq\left[\operatorname{Re}(\mathbf{x})^{T}, \operatorname{Im}(\mathbf{x})^{T}\right]^{T}, \overline{\mathbf{h}}_{i} \triangleq\left[\operatorname{Im}\left(b_{i}^{*} \mathbf{h}_{i}\right)^{T}, \operatorname{Re}\left(b_{i}^{*} \mathbf{h}_{i}\right)^{T}\right]^{T}$,

we can express the real and imaginary part of the transmitted signal in (6) as follows $\operatorname{Re}\left(b_{i}^{*} \mathbf{h}_{i}^{T} \mathbf{x}\right)=\overline{\mathbf{h}}_{i}^{T} \boldsymbol{\Pi}_{K} \overline{\mathbf{x}}, \operatorname{Im}\left(b_{i}^{*} \mathbf{h}_{i}^{T} \mathbf{x}\right)=\overline{\mathbf{h}}_{i}^{T} \overline{\mathbf{x}}$, where $\boldsymbol{\Pi}_{K} \triangleq\left[\mathbf{0}_{K, K}-\mathbf{I}_{K} ; \mathbf{I}_{K} \mathbf{0}_{K, K}\right]$ is a selection matrix such that $\mathbf{I}_{j}$, and $\mathbf{0}_{j, j}$ denotes the $j \times j$ identity matrix, and $j \times j$ 
zero matrix, respectively. Resolving the absolute value term in (6), we obtain two linear inequalities $\mathbf{t}_{2 i-1}^{T} \overline{\mathbf{x}} \geq \tilde{n}_{2 i-1}, \mathbf{t}_{2 i}^{T} \overline{\mathbf{x}} \geq \tilde{n}_{2 i}$, where $\mathbf{t}_{2 i-1}^{T} \triangleq-\overline{\mathbf{h}}_{i}^{T}+\tan \theta \overline{\mathbf{h}}_{i}^{T} \mathbf{\Pi}_{K}, \mathbf{t}_{2 i}^{T} \triangleq \overline{\mathbf{h}}_{i}^{T}+\tan \theta \overline{\mathbf{h}}_{i}^{T} \mathbf{\Pi}_{K}$, $\tilde{n}_{2 i-1} \triangleq \operatorname{Im}\left(b_{i}^{*} n_{i}\right)-\operatorname{Re}\left(b_{i}^{*} n_{i}\right) \tan \theta$, and $\tilde{n}_{2 i} \triangleq-\operatorname{Im}\left(b_{i}^{*} n_{i}\right)-$ $\operatorname{Re}\left(b_{i}^{*} n_{i}\right) \tan \theta$. The vectors $\mathbf{t}_{j}, j=1, \ldots, 2 K$, are deterministic and depend on the channel and the decision region defined by the angle $\theta$, and the scalars $\tilde{n}_{j}$ are real-valued Gaussian random variables (linear transformations of Gaussian random variables). Then the probability function in (5) can be written as a joint probability function $\operatorname{Pr}\left(\mathbf{t}_{2 i-1}^{T} \overline{\mathbf{x}} \geq \tilde{n}_{2 i-1}, \mathbf{t}_{2 i}^{T} \overline{\mathbf{x}} \geq \tilde{n}_{2 i}\right)$. Consider the bivariate standard normal probability distribution $\phi$ with zero mean such that $\phi(\mathbf{u} ; r)=\frac{1}{2 \pi \sqrt{1-r^{2}}} \exp \left(-\frac{1}{2} \mathbf{u}^{T} \boldsymbol{\Sigma}^{-1} \mathbf{u}\right)$ where $\mathbf{u} \triangleq\left[u_{1}, u_{2}\right]^{T}$, the correlation $r$ is defined as $r \triangleq \mathrm{E}\left\{\eta_{1} \eta_{2}\right\}$, with $|r|<1, \eta_{1}, \eta_{2}$ are the standardized random variables, i.e., $\mathrm{E}\left\{\left|\eta_{1}\right|^{2}\right\}=\mathrm{E}\left\{\left|\eta_{2}\right|^{2}\right\}=1$, and $\boldsymbol{\Sigma} \triangleq \mathrm{E}\left\{\left[\eta_{1}, \eta_{2}\right]^{T}\left[\eta_{1}, \eta_{2}\right]\right\}=\left[\begin{array}{ll}1 & r \\ r & 1\end{array}\right]$. The cumulative distribution function (CDF) of the standard bivariate normal distribution is defined by $\Phi(\mathbf{u} ; r)=\int_{-\infty}^{u_{1}} \int_{-\infty}^{u_{2}} \phi(\tilde{\mathbf{u}} ; r) d \tilde{u}_{1} d \tilde{u}_{2}$. Then the corresponding probability function for $u_{1} \geq \eta_{1}, u_{2} \geq \eta_{2}$ is given by $\operatorname{Pr}\left(u_{1} \geq \eta_{1}, u_{2} \geq \eta_{2}\right)=\Phi(\mathbf{u} ; r)$. Since $n_{i}$ is a circularly symmetric zero mean complex Gaussian random variable, we can conclude that $\tilde{n}_{j}$ is also a real-valued Gaussian with zero mean and variance $\sigma_{\tilde{n}}^{2} \triangleq \mathrm{E}\left\{\tilde{n}_{j}^{2}\right\}=\frac{\left(1+\tan ^{2} \theta\right) \sigma^{2}}{2}=\frac{\sigma^{2}}{2 \cos ^{2} \theta}$, i.e., $\tilde{n}_{j} \sim \mathcal{N}\left(0, \frac{\sigma^{2}}{2 \cos \theta^{2}}\right)$. Since $\tilde{n}_{2 i-1}$ and $\tilde{n}_{2 i}$ correspond to a real bivariate normal distribution, we can express the joint probability function as a joint normal CDF

$\operatorname{Pr}\left(\mathbf{t}_{2 i-1}^{T} \overline{\mathbf{x}} \geq \tilde{n}_{2 i-1}, \mathbf{t}_{2 i}^{T} \overline{\mathbf{x}} \geq \tilde{n}_{2 i}\right)=\Phi\left(\left[\frac{\mathbf{t}_{2 i-1}^{T} \overline{\mathbf{x}}}{\sigma_{\tilde{n}}}, \frac{\mathbf{t}_{2 i}^{T} \overline{\mathbf{x}}}{\sigma_{\tilde{n}}}\right]^{T} ; \bar{r}\right)$,

with the correlation of $\tilde{n}_{2 i-1}$ and $\tilde{n}_{2 i}$ is given by $\bar{r}=\frac{-1+\tan ^{2} \theta}{1+\tan ^{2} \theta}=$ $-\cos 2 \theta$. By (5) and (8), problem (4) can be reformulated as

$$
\begin{gathered}
\min _{\mathbf{x}, \rho} \rho \text { s.t. } 1-\Phi\left(\left[\frac{\mathbf{t}_{2 i-1}^{T} \overline{\mathbf{x}}}{\sigma_{\tilde{n}}}, \frac{\mathbf{t}_{2 i}^{T} \overline{\mathbf{x}}}{\sigma_{\tilde{n}}}\right]^{T} ; \bar{r}\right)-\rho \leq 0, \forall i, \\
\|\overline{\mathbf{x}}\|-\sqrt{P} \leq 0,\left\|\mathbf{B}_{l} \overline{\mathbf{x}}\right\|-\sqrt{\epsilon_{l}} \leq 0, \forall l,
\end{gathered}
$$

where $\mathbf{B}_{l} \triangleq\left[\begin{array}{cc}\operatorname{Re}\left(\mathbf{g}_{l}^{T}\right) & -\operatorname{Im}\left(\mathbf{g}_{l}^{T}\right) \\ \operatorname{Im}\left(\mathbf{g}_{l}^{T}\right) & \operatorname{Re}\left(\mathbf{g}_{l}^{T}\right)\end{array}\right]$ is a $2 \times 2 N$ real matrix. We remark that constraint $9 \mathrm{a}$ is generally non-convex. Note that the sufficient condition for the concavity of the standard bivariate normal $\mathrm{CDF}$ is non-trivial. Author in [9] showed that $\Phi(\mathbf{u} ; r)$ is concave in one variable under a certain condition on $u_{1}$ and $u_{2}$, respectively.

Lemma 1. [9] (Concavity in one variable) Let $-1 \leq r \leq 0$. Then $\Phi(\mathbf{u} ; r)$ is concave in $u_{i}$ for fixed $u_{j}$ with $j \neq i$, i.e., $\frac{\partial^{2} \Phi(\mathbf{u} ; r)}{\partial u_{i}^{2}} \leq 0$ for $i=1,2$, if

$$
u_{i} \geq \sqrt{\frac{\phi(1)}{2 \Phi(1)+\phi(1)}}, i=1,2,
$$

where the probability density function and CDF of a standard univariate normal distribution are given by

$$
\phi(u)=\frac{1}{\sqrt{2 \pi}} \exp \left(-\frac{u^{2}}{2}\right), \Phi(u)=\int_{-\infty}^{u} \phi(\tilde{u}) d \tilde{u},
$$

respectively.
In this paper, we further restrict the conditions on variables to guarantee the joint concavity of the CDF in $9 \mathrm{a}$ ) and show that these conditions are generally met in conventional transmission scenarios.

Theorem 1. (Joint Concavity) For $M \geq 4$, the standard bivariate normal CDF in (9a) is concave if $\mathbf{t}_{j}^{T} \overline{\mathbf{x}}$ satisfies the inequality

$$
\mathbf{t}_{j}^{T} \overline{\mathbf{x}} / \sigma_{\tilde{n}} \geq \alpha^{\star}(\bar{r}), j=1, \ldots, 2 K,
$$

with threshold $\alpha^{\star}(\cdot)$ denoting the optimal function value of the following constrained optimization problem:

$$
\alpha^{\star}(r): \min _{\alpha} \alpha \text { s.t. } \frac{\Phi\left(\alpha \frac{1-r}{\sqrt{1-r^{2}}}\right)}{\phi\left(\alpha \frac{1-r}{\sqrt{1-r^{2}}}\right)} \alpha \geq \frac{1-r}{\sqrt{1-r^{2}}} .
$$

\section{Proof. See Appendix}

Following from $9 \mathrm{a}$, we have

$$
\Phi\left(\frac{\mathbf{t}_{j}^{T} \overline{\mathbf{x}}}{\sigma_{\tilde{n}}}\right) \geq \Phi\left(\left[\frac{\mathbf{t}_{2 i-1}^{T} \overline{\mathbf{x}}}{\sigma_{\tilde{n}}}, \frac{\mathbf{t}_{2 i}^{T} \overline{\mathbf{x}}}{\sigma_{\tilde{n}}}\right]^{T} ; \bar{r}\right) \geq 1-\rho \geq 1-\rho^{\star},
$$

where $\rho^{\star}$ is the optimal value of $(9)$. If we assume that

$$
1-\rho^{\star} \geq \Phi\left(\alpha^{\star}(\bar{r})\right)
$$

then, by inequalities [14)-(15), and the strict monotonicity property of the standard univariate normal CDF, we ensure that condition (12) is satisfied. Thus, by Theorem 1, the assumption in (15) can guarantee problem (9) to be convex. That is, as of Theorem 1, for the optimal value $\rho^{\star}$ of $\left(9\right.$ such that $1-\Phi\left(\alpha^{\star}(\bar{r})\right) \geq \rho^{\star}$ for a given correlation $\bar{r}$, the optimization problem in 9 is convex. For example, when $M=4$, the value of $\rho^{\star}$ in (15) corresponds to a SEP of less than $30.64 \%$ which does not put any restrictions on our beamformer design as in typical applications much lower SEP values are required. Accordingly, the optimization problem in 9 is convex for all practical SEP constraints.

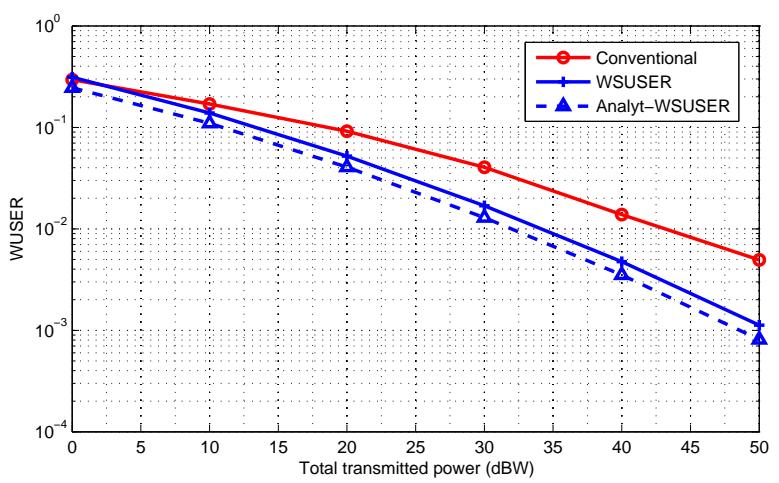

Fig. 2: WUSER performance versus power with $N=10, L=2$, $K=8, \epsilon_{l}=-2 \mathrm{dBW}$, and QPSK modulation.

\section{SIMULATIONS}

In this section, we present simulation results for a constructive interference-based downlink beamforming for CR network with $N=10$ antennas and $L=2$ PU. The system with $M$-PSK 

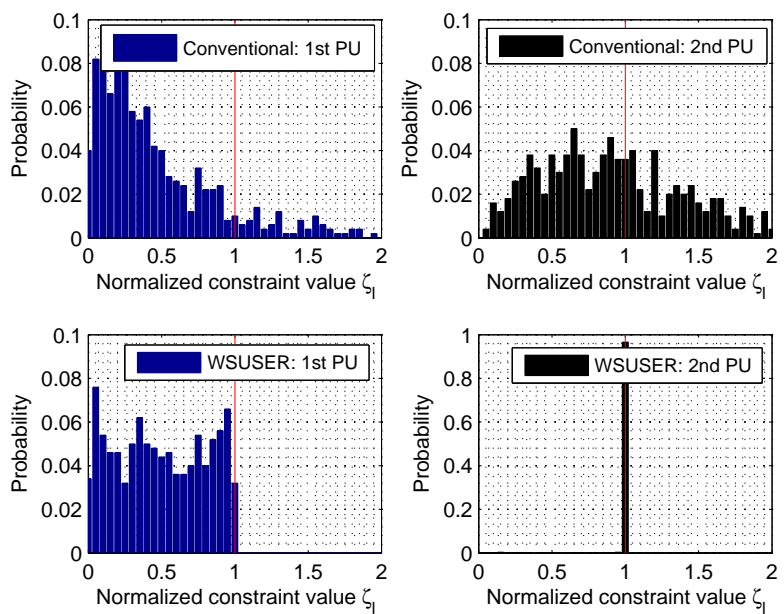

Fig. 3: Histogram of normalized constraint values $\zeta_{l}$ with $N=10$, $L=2, K=8, \epsilon_{l}=-2 \mathrm{dBW}, P=5 \mathrm{dBW}$, and QPSK modulation.

modulation is considered, i.e., $\theta=\pi / M$. A noise variance value of $\sigma^{2}=0.1$ is considered. In line with [20], we assume that the SUs and PUs connected to the SBS are located at directions $\omega_{1, \ldots, 10}=\left[3^{\circ}, 35^{\circ}, 10^{\circ}, 39^{\circ}, 17^{\circ}, 74^{\circ}, 24^{\circ}, 86^{\circ}, 30^{\circ}, 80^{\circ}\right]^{T}+\mathbf{r}_{1}$, $\tilde{\omega}_{1,2}=\left[50^{\circ}, 57^{\circ}\right]^{T}+\mathbf{r}_{2}$, where $\mathbf{r}_{1} \in \mathbb{C}^{10}$ and $\mathbf{r}_{2} \in \mathbb{C}^{2}$ are drawn from a uniform distribution in the interval $\left[-1^{\circ}, 1^{\circ}\right]$. Then the downlink channel from the SBS to $i$ th SU and $l$ th PU are modeled as [20]

$$
\begin{aligned}
& \mathbf{h}_{i}=\left[1, e^{j \pi \sin \omega_{i}}, \ldots, e^{j \pi(N-1) \sin \omega_{i}}\right]^{T}, \\
& \mathbf{g}_{l}=\left[1, e^{j \pi \sin \tilde{\omega}_{l}}, \ldots, e^{j \pi(N-1) \sin \tilde{\omega}_{l}}\right]^{T} .
\end{aligned}
$$

According to (2), we use the angle $\psi_{i}$ between the received signal $y_{i}$ and the transmitted symbol $b_{i}$ as an measure of the correct detection, which evaluates the performance of our proposed methods and the conventional method in [5 6]. The receive signal can be correctly detected if $\psi_{i}$ is within the interval $[-\theta, \theta]$. We introduce the normalized constraint value of interference power on an instantaneous basis $\zeta_{l}=\frac{\sum_{j=1}^{K} \sum_{i=1}^{K} b_{j}^{*} b_{i} \mathbf{w}_{j}^{\star} H \mathbf{g}_{l}^{*} \mathbf{g}_{l}^{T} \mathbf{w}_{i}^{\star}}{\epsilon_{l}}$, as an abstract measure of the constraint satisfaction to compare the performance of different methods [7]. The corresponding instantaneous interference power constraint at PU is satisfied if and only if $\zeta_{l} \leq 1$.

In Fig. 2. we fix the number of SUs and compare the performance of our proposed approaches and the conventional approach versus the total transmitted power $P$ for $K=8, \epsilon_{l}=-2 \mathrm{dBW}$, and QPSK modulation. It can be seen from Fig. 2 that the proposed approaches given in 9 outperform the conventional method in terms of the experimental WUSER performances. Notably, it can be observed in Fig. 2 that our analytic WUSER performance and lower bound of the computationally efficient approximate approach calculations match the experimental WUSER results of both (9). Furthermore, the computationally efficient approximate approach calculations match closely to the WSUSEP approach.

Fig. 3 depicts the histograms of normalized constraint values $\zeta_{l}$ with $K=8, \epsilon_{l}=-2 \mathrm{dBW}, P=5 \mathrm{dBW}$, and QPSK modulation. As can be observed from Fig. 3 the conventional technique only satisfies about $50 \%$ of the instantaneous interference power constraints for the second PU. This is due to the fact that the conventional method only considers the average interference power. However, our proposed approaches always satisfy the interference power constraints on an instantaneous basis. This consists of significant improvement over conventional $\mathrm{CR}$ beamformers which are prone to instantaneous PU outages.

\section{A. PROOF OF THEOREM 1}

To show the concavity, we need to use the first and second derivatives. It is well-known [9] that taking the first derivative with respect to $u_{1}$, we have $\frac{\partial \Phi(\mathbf{u}, r)}{\partial u_{1}}=\Phi\left(u_{2} \mid u_{1}\right) \phi\left(u_{1}\right)$, where the conditional distribution function $\Phi\left(u_{2} \mid u_{1}\right)$ is described by $\Phi\left(u_{2} \mid u_{1}\right)=$ $\Phi\left(\frac{u_{2}-r u_{1}}{\sqrt{1-r^{2}}}\right)$. Taking the second derivative with respect to $u_{1}$, we have $\frac{\partial^{2} \Phi(\mathbf{u} ; r)}{\partial u_{1}^{2}}=-r \frac{\partial^{2} \Phi(\mathbf{u} ; r)}{\partial u_{1} \partial u_{2}}-u_{1} \frac{\partial \Phi(\mathbf{u} ; r)}{\partial u_{1}}$. Similarly, we have $\frac{\partial^{2} \Phi(\mathbf{u} ; r)}{\partial u_{2}^{2}}=-r \frac{\partial^{2} \Phi(\mathbf{u} ; r)}{\partial u_{1} \partial u_{2}}-u_{2} \frac{\partial \Phi(\mathbf{u} ; r)}{\partial u_{2}}$.

By an abuse of notation, we redefine $\tilde{n}_{j}=\tilde{n}_{j} / \sigma_{\tilde{n}_{j}}$ and define $t_{j}(\overline{\mathbf{x}}) \triangleq \mathbf{t}_{j}^{T} \overline{\mathbf{x}} / \sigma_{\tilde{n}_{j}}$. Then $\tilde{n}_{j}$ is a standardized random variable for all $j$. To show the standard bivariate normal CDF in (9a) is concave, it is enough to prove that the Hessian matrix of the CDF

$$
\left[{\frac{\partial t_{2 i-1}}{\partial \overline{\mathbf{x}}}}^{T} \frac{\partial t_{2 i} T}{\partial \overline{\mathbf{x}}}\right] \mathbf{M}_{i}\left[{\frac{\partial t_{2 i-1}}{\partial \overline{\mathbf{x}}}}^{T}{\frac{\partial t_{2 i}}{\partial \overline{\mathbf{x}}}}^{T}\right]^{T},
$$

is a negative-semidefinite matrix where

$$
\mathbf{M}_{i} \triangleq\left[\begin{array}{cc}
\frac{\partial^{2} \Phi}{\partial t_{2 i-1}^{2}} & \frac{\partial^{2} \Phi}{\partial t_{2 i-1} \partial t_{2 i}} \\
\frac{\partial^{2} \Phi}{\partial t_{2 i-1} \partial t_{2 i}} & \frac{\partial^{2} \Phi}{\partial t_{2 i}^{2}}
\end{array}\right]
$$

The matrix in 18 is negative-semidefinite if the eigenvalues $\lambda_{i}^{ \pm}$of $\mathbf{M}_{i}$ are negative, which are equal to $\lambda_{i}^{ \pm}=\frac{\left(\frac{\partial^{2} \Phi}{\partial t_{2 i-1}^{2}}+\frac{\partial^{2} \Phi}{\partial t_{2 i}^{2}}\right) \pm \sqrt{\Delta_{i}}}{2}$, where $\Delta_{i} \triangleq\left(\frac{\partial^{2} \Phi}{\partial t_{2 i-1}^{2}}-\frac{\partial^{2} \Phi}{\partial t_{2 i}^{2}}\right)^{2}+4\left(\frac{\partial^{2} \Phi}{\partial t_{2 i-1} \partial t_{2 i}}\right)^{2}$. First the eigenvalues are real values as $\Delta_{i} \geq 0$. Second, we have $-1 \leq \bar{r} \leq 0$, for $M \geq 4$. Then, by (12) and Lemma $1 \mathrm{~B}$, we have $\frac{\partial^{2} \Phi}{\partial t_{j}^{2}} \leq 0$, which implies that $\left(\frac{\partial^{2} \Phi}{\partial t_{2 i-1}^{2}}+\frac{\partial^{2} \Phi}{\partial t_{2 i}^{2}}\right) \leq 0$. In order to show both eigenvalues are negative, we need to show that $-\left(\frac{\partial^{2} \Phi}{\partial t_{2 i-1}^{2}}+\frac{\partial^{2} \Phi}{\partial t_{2 i}^{2}}\right) \geq \sqrt{\Delta_{i}}$, which is equivalent to

$$
\frac{\partial^{2} \Phi}{\partial t_{2 i-1}^{2}} \frac{\partial^{2} \Phi}{\partial t_{2 i}^{2}} \geq\left(\frac{\partial^{2} \Phi}{\partial t_{2 i-1} \partial t_{2 i}}\right)^{2} .
$$

If

$$
-\frac{\partial^{2} \Phi}{\partial t_{2 i-1}^{2}} \geq \frac{\partial^{2} \Phi}{\partial t_{2 i-1} \partial t_{2 i}},-\frac{\partial^{2} \Phi}{\partial t_{2 i}^{2}} \geq \frac{\partial^{2} \Phi}{\partial t_{2 i-1} \partial t_{2 i}},
$$

then (20) holds. The inequalities in (21) are satisfied for $t_{2 i-1} \geq \alpha$, $t_{2 i} \geq \alpha$, if we have

$$
\frac{\Phi\left(\alpha \frac{1-\bar{r}}{\sqrt{1-\bar{r}^{2}}}\right)}{\phi\left(\alpha \frac{1-\bar{r}}{\sqrt{1-\bar{r}^{2}}}\right)} \alpha \geq \frac{1-\bar{r}}{\sqrt{1-\bar{r}^{2}}} .
$$

To find the minimum $\alpha$, we can solve the optimization problem in (13). Hence, for $t_{2 i-1} \geq \alpha^{\star}(\bar{r}), t_{2 i} \geq \alpha^{\star}(\bar{r})$, the inequalities in (21) hold. This completes the proof of the theorem. 


\section{B. REFERENCES}

[1] J. Mitola and G. Q. Maguire, "Cognitive radio: making software radios more personal," IEEE Personal Communications, vol. 6, no. 4, pp. 13-18, Aug 1999.

[2] Simon Haykin, "Cognitive radio: brain-empowered wireless communications," IEEE Journal on Selected Areas in Communications, vol. 23, no. 2, pp. 201-220, Feb 2005.

[3] Qing Zhao and B. M. Sadler, "A survey of dynamic spectrum access," IEEE Signal Processing Magazine, vol. 24, no. 3, pp. 79-89, May 2007.

[4] S. Sankaranarayanan, P. Papadimitratos, A. Mishra, and S. Hershey, "A bandwidth sharing approach to improve licensed spectrum utilization," in New Frontiers in Dynamic Spectrum Access Networks, 2005. DySPAN 2005. 2005 First IEEE International Symposium on, Nov 2005, pp. 279-288.

[5] Rui Zhang, Ying-Chang Liang, and Shuguang Cui, "Dynamic resource allocation in cognitive radio networks," IEEE Signal Processing Magazine, vol. 27, no. 3, pp. 102-114, May 2010.

[6] Xiao Fu, Jun Wang, and Shaoqian Li, "Joint power management and beamforming for base stations in cognitive radio systems," in Wireless Communication Systems, 2009. ISWCS 2009. 6th International Symposium on, Sept 2009, pp. 403 407.

[7] I. Wajid, M. Pesavento, Y.C. Eldar, and D. Ciochina, "Robust downlink beamforming with partial channel state information for conventional and cognitive radio networks," IEEE Transactions on Signal Processing, vol. 61, no. 14, pp. 3656-3670, July 2013.

[8] B.K. Chalise, S. ShahbazPanahi, A. Czylwik, and A.B. Gershman, "Robust downlink beamforming based on outage probability specifications," IEEE Transactions on Wireless Communications, vol. 6, no. 10, pp. 3498-3503, October 2007.

[9] András Prékopa, "On probabilistic constrained programming," Mathematical Programming Study, vol. 28, pp. 113-138, 1970.

[10] C. Masouros and E. Alsusa, "Soft linear precoding for the downlink of DS/CDMA communication systems," IEEE Transactions on Vehicular Technology, vol. 59, no. 1, pp. 203 215, Jan 2010

[11] C. Masouros and E. Alsusa, "Dynamic linear precoding for the exploitation of known interference in MIMO broadcast systems," IEEE Transactions on Wireless Communications, vol. 8, no. 3, pp. 1396-1404, March 2009.

[12] C. Masouros, "Correlation rotation linear precoding for MIMO broadcast communications," IEEE Transactions on Signal Processing, vol. 59, no. 1, pp. 252-262, Jan 2011.

[13] C. Masouros, M. Sellathurai, and T. Ratnarajah, "Interference optimization for transmit power reduction in TomlinsonHarashima precoded mimo downlinks," IEEE Transactions on Signal Processing, vol. 60, no. 5, pp. 2470-2481, May 2012.

[14] C. Masouros, M. Sellathurai, and T. Ratnarajah, "Interference optimization for transmit power reduction in TomlinsonHarashima precoded MIMO downlinks," IEEE Transactions on Signal Processing, vol. 60, no. 5, pp. 2470-2481, May 2012.

[15] C. Masouros, T. Ratnarajah, M. Sellathurai, C.B. Papadias, and A.K. Shukla, "Known interference in the cellular downlink: a performance limiting factor or a source of green signal power?," IEEE Communications Magazine, vol. 51, no. 10, pp. 162-171, October 2013.

[16] Gan Zheng, I. Krikidis, C. Masouros, S. Timotheou, D.-A. Toumpakaris, and Zhiguo Ding, "Rethinking the role of interference in wireless networks," IEEE Communications Magazine, vol. 52, no. 11, pp. 152-158, Nov 2014.

[17] C. Masouros and G. Zheng, "Exploiting known interference as green signal power for downlink beamforming optimization," IEEE Transactions on Signal Processing, vol. 63, no. 14, pp. 3628 - 3640, Jul 2015.

[18] M. Alodeh, S. Chatzinotas, and B. Ottersten, "Constructive multiuser interference in symbol level precoding for the MISO downlink channel," IEEE Transactions on Signal Processing, vol. 63, no. 9, pp. 2239-2252, May 2015.

[19] Ka Lung Law, Christos Masouros, Kai-Kit Wong, and Gan Zheng, "Constructive interference exploitation for QoS-based downlink beamforming optimization," submitted to IEEE Transactions on Signal Processing, 2015.

[20] Y. Huang and D.P. Palomar, "Rank-constrained separable semidefinite programming with applications to optimal beamforming," IEEE Transactions on Signal Processing, vol. 58, no. 2, pp. 664-678, Feb. 2010. 